\title{
Distributed Scheduling based on Multi-agent Systems and Optimization Methods
}

\author{
Filipe Alves ${ }^{1,2}$, Ana Maria A. C. Rocha ${ }^{2}$, Ana I. Pereira ${ }^{1,2}$, and Paulo Leitao ${ }^{1}$ \\ 1 Research Centre in Digitalization and Intelligent Robotics (CeDRI), Instituto \\ Politécnico de Bragança, Campus de Santa Apolónia, 5300-253 Bragança, Portugal \\ \{filipealves, apereira, pleitao\}@ipb.pt \\ 2 ALGORITMI Center, University of Minho, 4710-057 Braga, Portugal \\ arocha@dps.uminho.pt
}

\begin{abstract}
The increasing relevance of complex systems in dynamic environments has received special attention during the last decade from the researchers. Such systems need to satisfy products or clients desires, which, after accomplished might change, becoming a very dynamic situation. Currently, decentralized approaches could assist in the automation of dynamic scheduling, based on the distribution of control functions over a swarm network of decision-making entities. Distributed scheduling, in an automatic manner, can be answered by a service coordination architecture of the different schedule components. However, it is necessary to introduce the control layer in the solution, encapsulating an intelligent service that merge agents with optimization methods. Multi-agent systems (MAS) can be combined with several optimization methods to extract the best of the two worlds: the intelligent control, cooperation and autonomy provided by MAS solutions and the optimum offered by optimization methods. The proposal intends to test the intelligent management of the schedule composition quality, in two case studies namely, manufacturing and home health care.
\end{abstract}

Keywords: Scheduling - Multi-agent system · Optimization methods.

\section{Problem Statement}

Nowadays, sequencing, planning and scheduling are decision-making processes that play a crucial role in the manufacturing and service industries, economics and service operations management [8]. Moreover, the scheduling is a complex problem mainly due to its highly combinatorial aspect and its dynamic nature [4].

Traditionally, scheduling systems use centralized approaches, such as classical optimization methods, namely, heuristics, linear programming or metaheuristics. However, despite optimal solutions, they have high response times and consider the problems to be static and deterministic [6]. In contrast, multi-agent systems (MAS) offer an alternative way to design and control systems, differing from the conventional approaches due to fast and dynamic response and their inherent capabilities to adapt to emergence or disruptions [11]. However, the use of MAS, 
in turn, may not complement the search for optimized schedules provided by algorithms. Therefore, the lack of concrete solutions of distributed scheduling remains a problem, lacking validation on the design and implementation phase.

\section{Related Work}

Evolutions in Information and Communication Technologies (ICT) are based on the interaction of a multitude of different interconnected and even decisioncapable smart objects (belonging to industrial and/or logistics and operational systems), with associated information counterparts (agents, swarms, holons) or purely digital [9]. These emerging ICT concepts provide powerful new solutions to challenges not yet addressed using decentralized approaches to operational and distributed scheduling (DS) [12]. The scheduling visions can be supported by the latest achievements in MAS and optimization methods, that are being employed for the design and development of the distributed scheduling $[3,7]$.

\section{Hypothesis}

Based on the stated problem, a research question emerges: "How to provide faster and more dynamic solutions in DS systems without losing optimum?"

The hypothesis will be, merging the better of two worlds (MAS and optimization methods) will allow to achieve innovative optimal solutions ensuring dynamic responsiveness and providing to the organizations minimal external intervention. In addition, a swarm approach can be studied. The challenge is facing the natural inspiration and self-organization concepts in the design of scheduling solutions. The hypotheses to cooperate with entities that can be regulated by simple rules, with reactive behavior and interactions in an environment without central authority, potentiates a DS architecture [5].

\section{Proposal}

This section outlines the vision of creating an architecture that facilitates an automatic search, selects, composes and provides DS solutions. Considering the problem, requirements and objectives, in this work a MAS approach is proposed taking into consideration recursive swarm design principles, that naturally matches the DS structure. Each agent is responsible to collect and analyze the data from its connected software, to support the control and monitoring actions. In addition, each agent has the ability to cooperate with other agents in order to achieve a balance between the optimums. The generic internal agent architecture illustrated in Figure 1 comprises the intelligent management, the communication module (inter-agent), the interface module (intra-agent) and the auxiliary modules, which the user can perform transparently.

Each agent is endowed with local autonomy and intelligence required to dynamically adapt to changes in the system. In this sense, the intelligent management module defines the several functions that are related to the acquisition and 


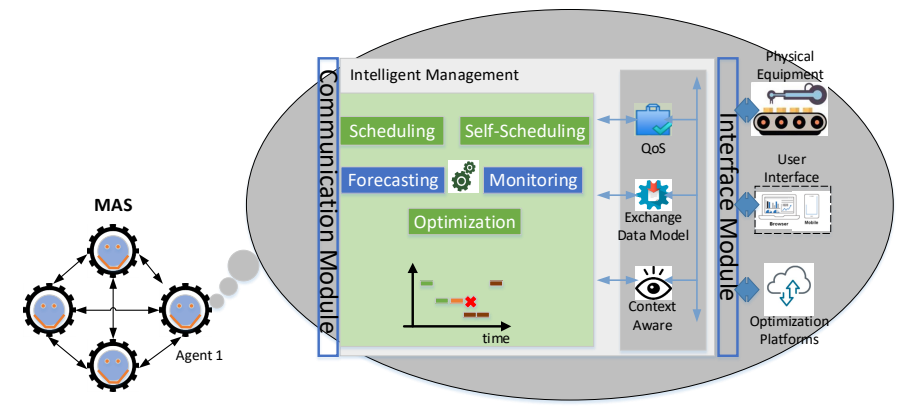

Fig. 1. Generic internal architecture of an agent.

analysis of the distributed scheduling, comprising: scheduling, forecasting, selfscheduling, monitoring and optimization. The interface module provides, when needed, a connection to the physical devices, optimization platforms and/or displays information to the users using a friendly graphical user interface, which allows real-time monitoring of the scheduling state. These functions, as part of the intelligent management component, are complemented by the quality of service, exchange data model and context-aware modules.

\section{Preliminary Results and/or Evaluation Plan}

The proposal will be applied to two different domains: in the manufacturing and in the Home Health Care (HHC).The manufacturing domain refers to the case study of a flexible manufacturing system production cell "AIP PRIMECA" [10]. In this sense, an approach and simulation has already been developed in this context, but using a simple dataset [1]. On the other hand, HHC services have significantly increased, namely in Portugal. The main idea is to find the schedule for home care visits for a certain day or days in a period horizon. A simulation has been carried out involving a real case study of a Health Unit in Bragança subject to unexpected events [2].

The simulations have benefited from a flexible architecture in obtaining optimized solutions providing an improvement in its usefulness. In turn, it was possible to offer, task effort distribution, reduced impact of broken resources and finally, fastest and most accurate reaction to changes or disruptions by agents. However, the scheduling comes from a centralized module, which in turn controls the simulation. The future goal will be to benefit from a distributed architecture for flexible scheduling solutions balancing the simple and fast reactions of the agents with the solutions by optimization methods to improve the utility.

\section{Reflections}

This paper points out and discusses the distributed scheduling problem highlighting the benefits of combining it with a swarm approach, MAS and optimization 
methods. To address such challenge, two major directions were explored. The first direction concerns the service-oriented multi-agent system swarm approach, to intelligently and dynamically select the most appropriate applications provided by reliable entities to increase the confidence and quality of the needed scheduling composition. Applications will be especially subject to reactive environments with self-scheduling challenges and decentralized decisions. The second direction consists of the combination of MAS with optimization methods for collaboration of optimized scheduling and thus obtain support for group decision-making.

In conclusion, the proposal can explore multidisciplinary domains. The applicability and optimization in different applications, such as manufacturing and home health care, can prove the dynamics in domains with emerging needs.

Acknowledgments. This work has been supported by FCT - Fundação para a Ciência e Tecnologia within the Projects Scope: UID/CEC/00319/2019.

\section{References}

1. Alves, F., Varela, M.L.R., Rocha, A.M.A.C., Pereira, A.I., Barbosa, J., Leitão, P.: Hybrid system for simultaneous job shop scheduling and layout optimization based on multi-agents and genetic algorithm. $18^{\text {th }}$ International Conference on Hybrid Intelligent Systems (HIS 2018), Springer (In press), Portugal (2018)

2. Alves, F., Pereira, A.I., Barbosa, J., Leitão, P.: Scheduling of home health care services based on multi-agent systems. In: Highlights of Practical Applications of Agents, Multi-Agent Systems, and Complexity: The PAAMS Collection. pp. 12-23. Springer International Publishing, Cham (2018)

3. Çaliş, B., Bulkan, S.: A research survey: review of ai solution strategies of job shop scheduling problem. Journal of Intelligent Manufacturing 26(5), 961-973 (2015)

4. Gen, M., Lin, L.: Multiobjective evolutionary algorithm for manufacturing scheduling problems: state-of-the-art survey. Journal of Intelligent Manufacturing 25(5), 849-866 (Oct 2014). https://doi.org/10.1007/s10845-013-0804-4

5. Leitão, P., Barbosa, J.: Adaptive scheduling based on self-organized holonic swarm of schedulers. In: 2014 IEEE 23rd International Symposium on Industrial Electronics (ISIE). pp. 1706-1711 (June 2014). https://doi.org/10.1109/ISIE.2014.6864872

6. Leitão, P., Restivo, F.: A holonic approach to dynamic manufacturing scheduling. Robotics and Computer-Integrated Manufacturing 24(5), 625 - 634 (2008)

7. Ouelhadj, D., Petrovic, S.: A survey of dynamic scheduling in manufacturing systems. Journal of Scheduling 12(4), 417 (Oct 2008)

8. Pinedo, M.L.: Scheduling: theory, algorithms, and systems. Springer (2016)

9. Trentesaux, D., Borangiu, T., Thomas, A.: Emerging ict concepts for smart, safe and sustainable industrial systems (2016)

10. Trentesaux, D., Pach, C., Bekrar, A., Sallez, Y., Berger, T., Bonte, T., Leitão, P., Barbosa, J.: Benchmarking flexible job-shop scheduling and control systems. Control Engineering Practice 21(9), 1204 - 1225 (2013)

11. Wooldridge, M.: An Introduction to MultiAgent Systems. Wiley Publishing, 2nd edn. (2009)

12. Yang, Q., Yang, T., Li, W.: Smart Power Distribution Systems: Control, Communication, and Optimization. Elsevier Science (2018) 